\title{
A systematic study on latent semantic analysis model parameters for mining biomedical literature Mohammed Yeasin ${ }^{1}$, Haritha Malempati*1, Ramin Homayouni ${ }^{2}$ and Mohammad Shahed Sorower ${ }^{1}$
}

\author{
Address: ${ }^{1}$ Department of Electrical and Computer Engineering, University of Memphis, Memphis, TN 38111, USA and ${ }^{2}$ Bioinformatics Program, \\ University of Memphis, Memphis, TN 38111, USA \\ Email: Haritha Malempati* - hmalempt@memphis.edu \\ * Corresponding author
}

from UT-ORNL-KBRIN Bioinformatics Summit 2009

Pikeville, TN, USA. 20-22 March 2009

Published: 25 June 2009

BMC Bioinformatics 2009, I 0(Suppl 7):A6 doi:10.1 186/147|-2105-10-S7-A6

This abstract is available from: http://www.biomedcentral.com/I47I-2/05/I0/S7/A6

(C) 2009 Yeasin et al; licensee BioMed Central Ltd.

\section{Background and rationale}

Latent semantic analysis (LSA) is considered to be an efficient text mining technique [1] but most approaches developed on this paradigm are based on adhoc principles. A systematic study on the parameters affecting the performance of LSA is expected to provide guidelines to objectively select the LSA model parameters in a way that is consistent with the data and the application. In this study, empirical analyses were conducted using a previously published 50 gene data set [2] to examine the effects of the following parameters (outlined in Figure 1): Parameters are: (i) stemming, stop-words and word counts (to discard abstract with not enough information), (ii) corpus content (e.g., abstracts with and without titles), (iii) inclusion or exclusion of the dc component or $1^{\text {st }}$ Eigen vector (that adds bias to the model), (iv) objective criteria to choose the number of factors (Eigen vectors) to create the model, (v) information theoretic criteria to select features (words in the corpus) instead of considering complete set of features.

\section{Methodology}

Two datasets, one with titles and abstracts and the other with only abstracts were used to conduct empirical analyses. Preprocessing steps included stemming, stop word removal, as well as removal of documents with less than 100 terms. The term frequency-inverse document frequency (TF-IDF) matrix of size $8714^{*} 50$ was constructed using the dataset. Singular value decomposition (SVD) on the TF-IDF matrix was used to compute the encoding of the dataset and only $k$ components were retained based on the following objective criteria:

1. Top 25 Eigen vectors

$$
\text { 2. } \frac{\Sigma \sigma_{p}^{2}}{\Sigma \sigma_{n}^{2}} \sim 97 \% \text {, }
$$

$\Sigma \sigma_{p}^{2}:$ energy content within p Eigen vectors,

$\Sigma \sigma_{n}^{2}:$ Energy content with $\mathrm{n}$ (all) Eigen vectors

3. $\sigma_{k} \sim \frac{0.7}{n}$

$\mathrm{n}$ : number of documents, k: indices of Eigen vector, S: singular value

In addition, the effect of bias was studied by excluding the $1^{\text {st }}$ Eigen vector (dc component).

Different combinations of these parameters were studied and the performance of various LSA models was evaluated by determining the average precision, recall values. The 


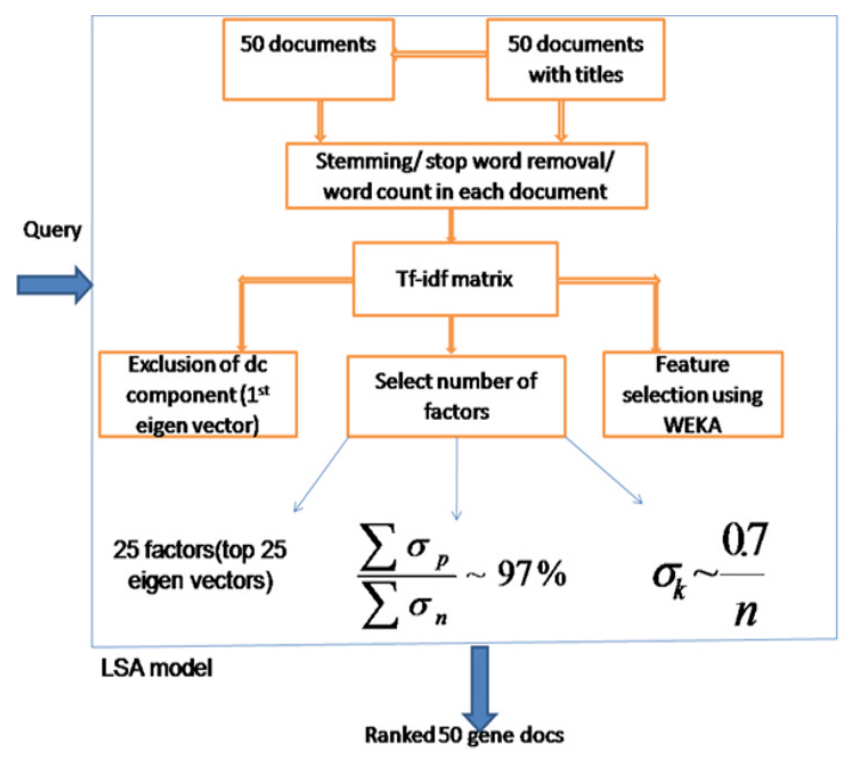

Figure I

Illustration of the methodology.

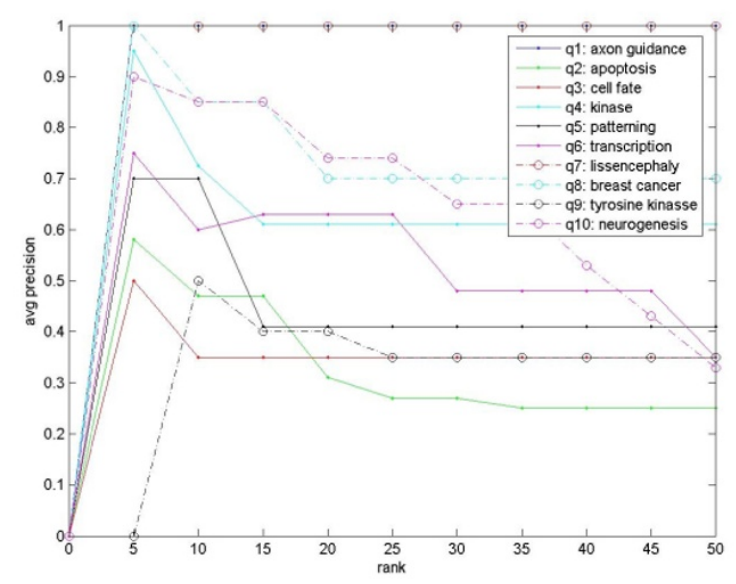

Figure 2

Average precision vs. rank curve. A combination of three parameters was used: inclusion of titles, exclusion of ${ }^{\text {st }}$ Eigen vector and $0.7 / \mathrm{n}$ objective criterion for factor selection. This combination provides better performance than with individual parameters.

Table I: Average precision values of queries (rows) across different parameters (columns)

\begin{tabular}{|c|c|c|c|c|c|c|c|}
\hline & $\begin{array}{c}\text { Abstract } \\
s\end{array}$ & Titles \& Abstracts & 25 Eigen vectors & $\begin{array}{l}97 \% \text { energy } \\
\text { criterion }\end{array}$ & $0.7 / \mathrm{n}$ criterion & Feature selection & No Ist Eigen vector \\
\hline axon guidance & 0.33 & 1 & 1 & 1 & 1 & 1 & 1 \\
\hline apoptosis & 0.2 & 0.20 & 0.18 & 0.19 & 0.23 & 0.3 & 0.27 \\
\hline cell fate & 0.24 & 0.42 & 0.3 & 0.38 & 0.4 & 0.37 & 0.4 \\
\hline kinase & 0.6 & 0.78 & 0.6 & 0.62 & 0.75 & 0.79 & 0.65 \\
\hline patterning & 0.35 & 0.46 & 0.4 & 0.42 & 0.5 & 0.52 & 0.46 \\
\hline transcription & 0.3 & 0.38 & 0.32 & 0.37 & 0.47 & 0.4 & 0.39 \\
\hline lissencephaly & 0.4 & 1 & 1 & I & 1 & 1 & 1 \\
\hline breast cancer & 0.25 & 0.74 & 0.37 & 0.5 & 0.75 & 0.67 & 0.7 \\
\hline tyrosine kinase & 0.1 & 0.3 & 0.12 & 0.15 & 0.3 & 0.28 & 0.28 \\
\hline neurogenesis & 0.18 & 0.32 & 0.13 & 0.18 & 0.32 & 0.3 & 0.3 \\
\hline
\end{tabular}

best model is defined as the one with relatively high average precision across a set of varied queries.

\section{Results and conclusion}

Performance analysis (average precision-recall curves, Fmeasure etc.) using Gene Ontology classifications corresponding to the 50 gene collection show that not all parameters significantly affect the performance of LSA model (Table 1). In general, adding titles in addition to the abstracts substantially increased the average precision. In addition, using $0.7 / \mathrm{n}$ criteria produced better results than using 25 Eigen vectors or the $97 \%$ criteria. It was found that the best performance was achieved by combining 3 parameters: inclusion of title in abstracts in the corpus, exclusion of the dc component, and selection of Eigen vectors based on objective criterion (Figure 2). This work provides a framework for determining the best parameters in using LSA for ranking genes with respect to queries. Future work will focus on evaluating this framework using different gene document collections.

\section{References}

I. Vanteru BC, Shaik JS, Yeasin M: Semantically linking and browsing PubMed abstracts with gene ontology. BMC Genomics 2008, 9:S10.

2. Homayouni R, Heinrich K, Wei L, Berry M: Gene clustering by latent semantic indexing of MEDLINE abstracts. Bioinformatics 2005, 21 ( ( ): 104. 